\title{
Expression of serum AMPD1 in thyroid carcinoma and its clinical significance
}

\author{
TIANZHOU ZHA ${ }^{1,2}$ and HAORONG WU ${ }^{1}$ \\ ${ }^{1}$ Department of General Surgery, The Second Affiliated Hospital of Soochow University, Suzhou, Jiangsu 215004; \\ ${ }^{2}$ Department of General Surgery, The Affiliated Yixing Hospital of Jiangsu University, Yixing, Jiangsu 214200, P.R. China
}

Received November 13, 2017; Accepted January 5, 2018

DOI: $10.3892 /$ etm.2018.5859

\begin{abstract}
This study investigated the expression of adenosine monophosphate deaminase 1 (AMPD1) in serum of patients with papillary thyroid carcinoma (PTC) and its clinical significance. The expression levels of AMPD1 mRNA in serum of 157 patients with PTC and 100 normal controls were detected by real-time fluorescent quantitative polymerase chain reaction (PCR), and the relationships between expression level of AMPD1 in serum of PTC patients and clinicopathological factors as well as prognosis were analyzed. The results of realtime fluorescent quantitative PCR showed that the expression of AMPD1 mRNA in serum of PTC patients was lower than that in normal human serum $(\mathrm{P}<0.01)$. The expression of AMPD1 in serum of PTC patients was not significantly different from the clinicopathological features such as sex, age, lymph node metastasis and the number of lesions $(\mathrm{P}>0.05)$; there were distinct differences between its expression and tumor-nodemetastasis $(\mathrm{TNM})$ staging and tumor diameter $(\mathrm{P}<0.05)$. The single factor Cox analysis revealed that sex, age, number of lesions, TNM staging and the occurrence of lymph node metastasis were significantly correlated with the prognosis of patients $(\mathrm{P}<0.05)$. Multivariate Cox analysis showed that TNM staging hazard ratio $(\mathrm{HR})=2.93,95 \%$ confidence interval (CI): $1.52-7.04, \mathrm{P}=0.015$ was an independent prognostic factor in PTC patients. Survival analysis indicated that there was a statistically significant difference in the 5-year overall survival rate between patients with high expression of AMPD1 and those with low expression $(\mathrm{P}=0.007)$. In conclusion, the expression of AMPD1 in serum of patients with PTC is closely related to the malignant evolution of PTC and clinical prognosis of patients. AMPD1 is expected to become an important molecule in judging the clinical prognosis of PTC patients, and may become a new target for molecular targeted therapy of PTC.
\end{abstract}

Correspondence to: Dr Haorong Wu, Department of General Surgery, The Second Affiliated Hospital of Soochow University, 1055 Sanxiang Road, Gusu, Suzhou, Jiangsu 215004, P.R. China E-mail:wu_haorong1@163.com

Key words: serum AMPD1, thyroid carcinoma

\section{Introduction}

Thyroid cancer represents the most common cancer in the endocrine system (1-4). Most thyroid cancers originate from thyroid follicular cells (>90\%), which can differentiate into papillary thyroid carcinoma (PTC) and follicular thyroid carcinoma (FTC), while only less than 5\% derive from C-cells (5). Of the PTC patients $40-60 \%$ are aged over 45 years, mainly middle aged and elderly people with lymphatic metastasis (6). The most common follicular tumors now are mostly benign hyperplastic tumors, and PTC represents the most common thyroid cancer (approximately 90\%) (7). PTC and FTC may progress to a poorly differentiated carcinoma or complete loss of differentiation to produce anaplastic thyroid carcinoma (ATC) (8).

A large number of studies have been conducted to screen candidate markers of thyroid cancer at present. The differential expression of excessively large numbers of molecules was screened out in thyroid carcinoma tissues (9-11). The adenosine monophosphate deaminase (AMPD) encoded by AMPD1 gene has the function of catalyzing adenosine monophosphate inosinemonpho sphate $(12,13)$ in purine nucleotide metabolism and energy metabolism, especially skeletal muscle and cardiac muscle. Currently, it is known that AMPD1 gene is generally localized on the p13-21 region chromosome, with a length of $20 \mathrm{~kb}$, consisting of 16 exons and 15 introns (14). In most cases, the presence of the nonsense mutation of 34 at position of AMPD1 and common polymorphism of C-T transformation contribute to the emergence of premature termination codon; some related metabolic muscle disorders are caused by the lack of AMPD activity (15). It is proved that mutations in the AMPD1 allele play a protective role in the onset of congestive heart failure, which can also help (16) and relatively reduce aortic stiffness and inflammation in patients with coronary artery disease (CAD) (13). However, there are few studies on AMPD1 in human malignant tumors; particularly, its relationship with the occurrence and development of thyroid cancer, and the expression level and prognosis of thyroid cancer are rarely reported. This study aimed to further understand the expression of AMPD1 and its relationship with cancer.

\section{Materials and methods}

Clinical data. A total of 157 patients with PTC diagnosed by pathology admitted to the Department of Oncology in 
Table I. Internal reference of AMPD1 primers.

\begin{tabular}{lc}
\hline Gene & \multicolumn{1}{c}{ Primer sequence } \\
\hline AMPD1 & U: 5'-AAGGCCGCCCAGAGCTTATTCAT-3' \\
primer & D: 5'-CTTCAGCAGGGTCCGAGGTATTC-3' \\
U6 internal & U: 5'-CTCGCTTCGGCAGCACA-3' \\
reference & D: 5'-AACGCTTCACGAATTTGCGT-3' \\
\hline
\end{tabular}

$\mathrm{U}$, upstream primer; $\mathrm{D}$, downstream primer.

The Second Affiliated Hospital of Soochow University from May 2011 to August 2016 were collected, including 75 males and 82 females, aged 43-77 years, with an average age of $62.15 \pm 10.61$ years. According to the eighth edition of tumor-node-metastasis (TNM) staging issued by American Joint Committee on Cancer (AJCC), there were 44 cases in stage I, 63 cases in stage II, 32 cases in stage III and 18 cases in stage IV. One hundred normal people were enrolled as control group. None of the patients had been treated with radiotherapy and chemotherapy before operation. The study was approved by the Ethics Committee of The Second Affiliated Hospital of Soochow University, and patients signed the informed consent.

Main instruments and reagents. ABI StepOne Plus (Applied Biosystems, Foster City, CA, USA) fluorescence quantitative polymerase chain reaction (PCR) instrument, NanoDrop 2000 spectrophotometer, KH19A desktop high-speed highperformance centrifuge (KAIDA); $-80^{\circ} \mathrm{C}$ low-temperature refrigerator (Thermo Fisher Scientific); AxyGen Total RNA Extraction kit was purchased from Takara Biotechnology Co., Ltd., Dalian, China; reverse transcription kit was from Thermo Fisher Scientific; fluorescent quantitative PCR kit was from Takara Biotechnology Co., Ltd.

Collection of samples. Peripheral blood $(5 \mathrm{ml})$ was extracted from patients with PTC and volunteers in normal control group before operation and placed into an ethylenediaminetetraacetic acid-k (EDTA-k) anticoagulant tube, followed by centrifugation at $3500 \mathrm{x} \mathrm{g}$ for $5 \mathrm{~min}$. The supernatant was taken and centrifuged at $8000 \mathrm{xg}$ at $4^{\circ} \mathrm{C}$ for $10 \mathrm{~min}$. The supernatant was collected and stored at $-80^{\circ} \mathrm{C}$.

Design and synthesis of primers. cDNA sequences of AMPD1 gene were synthesized using primer design software Primer 5, and primers were synthesized by Guangzhou Shangeng Biotechnology Co., Ltd. (Table I). PCR amplification reac-

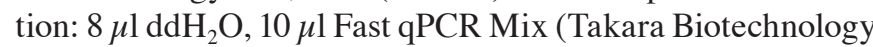
Co., Ltd.), $2 \mu \mathrm{l}$ cDNA template, $0.2 \mu \mathrm{l}$ upstream primer and $0.2 \mu \mathrm{l}$ downstream primer $(10 \mu \mathrm{mol} / \mathrm{l})$; PCR was amplified by Applied Biosystems StepOne Plus: pre-denaturation at $95^{\circ} \mathrm{C}$ for $10 \mathrm{~min}$, denaturation at $95^{\circ} \mathrm{C}$ for $15 \mathrm{sec}$, annealing and extending at $60^{\circ} \mathrm{C}$ for $30 \mathrm{sec}$, a total of 45 cycles. The relative expression level of AMPD1 was calculated using $2^{-\Delta C T}$ method.

Statistical analysis. SPSS 22.0 (Version X; IBM, Armonk, NY, USA) statistical software was used for statistical analysis.

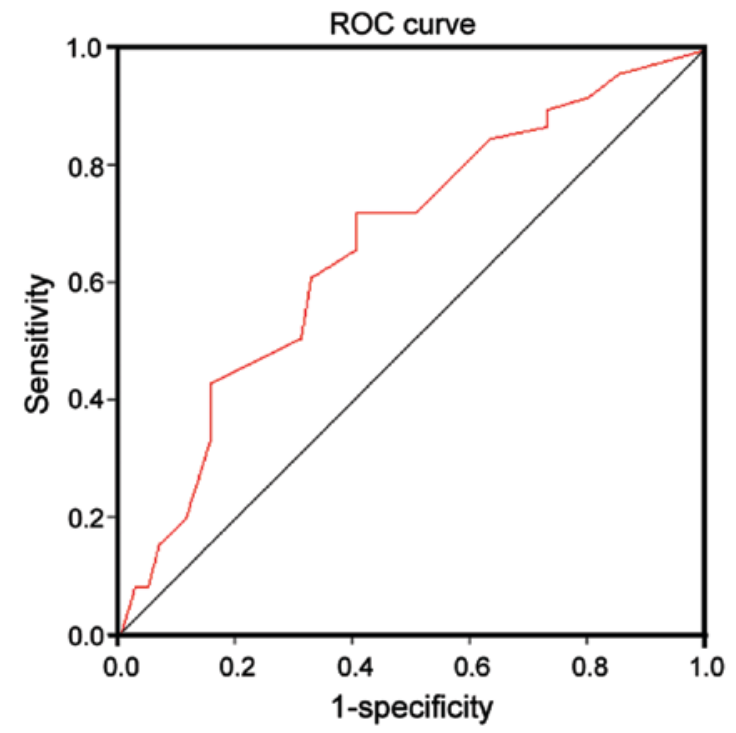

Figure 1. ROC curves of AMPD1 in serum of PTC and normal control group. The value of AMPD1 is high in predicting risk of PTC, AUC is 2.93 and $95 \%$ $\mathrm{CI}$ is $1.52-7.04$.

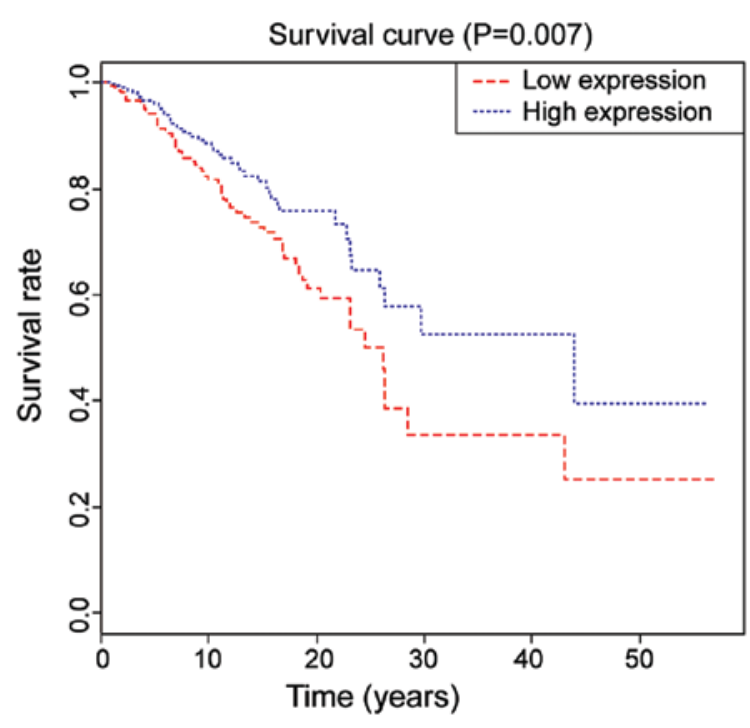

Figure 2. Kaplan-Meier survival curve of PCT patients in high or low expression group of AMPD1. The 5-year survival rate in the group with low expression of AMPD1 is lower than that in group with high expression of AMPD1 $(\mathrm{P}=0.007)(\mathrm{P}<0.05)$.

Measurement data were expressed as mean $\pm \mathrm{SD}$, and t-test was applied for the comparison of means between two samples. Pearson's correlation analysis was utilized for analyzing the correlation. Furthermore, the relationship between AMPD1 and 5-year survival rate was analyzed by single-factor and multivariate Cox regression. $\mathrm{P}<0.05$ was considered to indicate a statistically significant difference.

\section{Results}

Expression levels of AMPDI in PTC patients and control group. The results of expression levels of AMPD1 in serum of PTC patients and control group detected by real-time reverse transcription polymerase chain reaction (RT-PCR) indicated 
Table II. Expression level of AMPD1 in serum of PTC patients and control group.

\begin{tabular}{lcccc}
\hline Group & $\mathrm{n}$ & $\begin{array}{c}\text { Expression level } \\
\text { of AMPD1 }\end{array}$ & P-value & t value \\
\hline Patient & 157 & $2.14 \pm 2.45$ & & \\
Control & 100 & $4.67 \pm 1.91$ & $<0.01$ & 5.925 \\
\hline
\end{tabular}

Table III. Relationships between expression level of AMPD1 and clinicopathological features in PTC patients.

\begin{tabular}{lll}
\hline $\begin{array}{l}\text { Clinical pathological } \\
\text { features }\end{array}$ & $\begin{array}{c}\text { Expression level } \\
\text { of AMPD1 }\end{array}$ & P-value \\
\hline $\begin{array}{l}\text { Age/years } \\
\geq 45\end{array}$ & $2.15(1.65-3.03)$ & 0.059 \\
$<45$ & $3.81(1.25-2.96)$ & \\
Sex & & 0.751 \\
Male & $3.67(1.86-2.55)$ & \\
Female & $3.11(1.57-2.67)$ & \\
Tumor diameter & & 0.045 \\
$\geq 20$ mm & $2.01(1.29-2.59)$ & \\
$<20$ mm & $3.54(1.89-3.09)$ & \\
Lymph node metastasis & & 0.135 \\
Yes & $2.39(1.48-3.21)$ & \\
No & $3.18(1.80-2.67)$ & \\
TNM staging & & 0.017 \\
I-II & $3.44(1.36-3.54)$ & \\
III-IV & $1.87(1.22-2.57)$ & \\
Number of lesions & & \\
Single & $2.16(1.69-3.49)$ & \\
Multiple & $1.54(2.19-3.56)$ & \\
\hline
\end{tabular}

that the expression level of AMPD1 in serum of PTC patients was remarkably reduced compared with that in normal control group $(\mathrm{P}<0.01)$ (Table II). The receiver operating characteristic (ROC) curve analysis was further performed, suggesting that the risk in PTC patients could be predicted by AMPD1 with a certain value, the area under the curve (AUC) was 0.713 , and $95 \%$ confidence interval (CI) was $0.692-0.797$ (Fig. 1).

Relationships between expression level of AMPD1 and clinicopathological features in PTC patients. According to the differential expression of AMPD1 in PTC patients and combination with clinical pathological data, it was found that the expression of AMPD1 in serum of PTC patients was not significantly different from the clinicopathological features such as sex, age, lymph node metastasis and the number of lesions $(\mathrm{P}>0.05)$; there were distinct differences between its expression and TNM staging and tumor diameter $(\mathrm{P}<0.05)$ (Table III).
Table IV. Single factor analysis for PTC patients.

\begin{tabular}{|c|c|c|c|c|}
\hline Influencing factors & $\mathrm{n}$ & $\begin{array}{c}5 \text {-year survival } \\
\text { rate }(\%)\end{array}$ & HR & P-value \\
\hline Sex & & & 0.77 & 0.036 \\
\hline Male & 75 & 60.6 & & \\
\hline Female & 82 & 69.4 & & \\
\hline Age & & & 0.84 & 0.574 \\
\hline$\geq 45$ & 69 & 64.7 & & \\
\hline$<45$ & 88 & 60.1 & & \\
\hline Tumor diameter & & & 1.91 & 0.617 \\
\hline$\geq 20 \mathrm{~mm}$ & 61 & 50.3 & & \\
\hline$<20 \mathrm{~mm}$ & 96 & 63.4 & & \\
\hline Lymph node metastasis & & & 0.67 & 0.007 \\
\hline Yes & 49 & 55.6 & & \\
\hline No & 108 & 67.4 & & \\
\hline TNM staging & & & 0.52 & 0.057 \\
\hline I-II & 85 & 68.1 & & \\
\hline III-IV & 72 & 50.2 & & \\
\hline Number of lesions & & & & 0.011 \\
\hline Single & 79 & 67.4 & & \\
\hline Multiple & 78 & 52.7 & & \\
\hline
\end{tabular}

Table V. Multivariate analysis for prognosis of PTC patients.

\begin{tabular}{lccc}
\hline Influencing factor & HR & P-value & 95\% CI \\
\hline Sex & 0.69 & 0.547 & $1.27-5.17$ \\
Lymph node metastasis & 0.81 & 0.095 & $1.47-4.94$ \\
TNM staging & 2.93 & 0.015 & $1.52-7.04$ \\
Number of lesions & 1.27 & 0.184 & $1.38-6.14$ \\
\hline
\end{tabular}

Analysis of prognosis-related factors in PTC patients. The 5-year survival rate in PTC patients with high expression of AMPD1 was 62.4, which was 45.5 in the group with low expression of AMPD1, and the difference was statistically significant ( $\mathrm{P}=0.007$, Fig. 2). The single factor Cox analysis revealed that sex, age, number of lesions, TNM staging and the occurrence of lymph node metastasis were significantly correlated with the prognosis of patients $(\mathrm{P}<0.05)$ (Table IV). Multivariate Cox analysis showed that TNM staging hazard ratio $(\mathrm{HR})=2.93,95 \% \mathrm{CI}: 1.52-7.04, \mathrm{P}=0.015$ was an independent prognostic factor in PTC patients (Table V).

\section{Discussion}

Thyroid cancer (THCA) is a common malignant tumor in the endocrine system, which mainly derives from thyroid epithelial cells, accounting for $1 \%$ of the systemic malignancy. Currently, its incidence is increasing year by year. Papillary thyroid carcinoma (PTC) is the most common pathological type of THCA, and the cervical lymph node metastasis 
may appear in the early stage of the disease. Thus, the early diagnosis and surgical treatment of PTC are conducive to improving the prognosis of patients (17).

The adenosine monophosphate deaminase (AMPD) encoded by AMPD1 gene has the function of catalyzing adenosine monophosphate inosinemonophosphate $(8,9)$ in purine nucleotide metabolism and energy metabolism, especially skeletal muscle and cardiac muscle. At present, AMPD1 gene has been studied in diabetes mellitus (18) and cardiovascular disease (19). The purpose of this study is to identify new tumor markers in THCA thus controlling the disease through better diagnosis and treatment.

The study revealed that the expression level of AMPD1 in serum of PTC patients was significantly lower than that of normal human serum; by combining with clinical analysis, it showed that AMPD1 expression was positively related with age, tumor diameter and TNM staging of patients; the higher the age was, the lower the expression level of AMPD1 would be; the larger the tumor diameter was, the lower the expression level of AMPD1 would be; the higher the TNM staging was, the lower the expression level of AMPD1 would be. The above results indicated that AMPD1 may be involved in the pathological development of PTC.

This study revealed that the risk of PTC can be predicted by AMPD1 expression, which is correlated with pathological staging, TNM grading, distant metastasis and lymph node metastasis of patients. Importantly, the low expression of AMPD1 is associated with low overall survival rate, which is an independent prognostic factor for PTC patients. Clinically, it is difficult to diagnose papillary thyroid microcarcinoma (PTMC) with diameter less than $10 \mathrm{~mm}$ before surgery due to the small size, unobvious onset and lack of specific clinical manifestations and it is only found when thyroidectomy or progressive lymph node metastasis is required due to other causes (20).

In conclusion, this study indicated that AMPD1 is closely linked to TNM staging. In the following study, we will further expand the sample size to analyze the expression of AMPD1 in PTC, so as to provide a basis for the diagnosis of PTC. In summary, AMPD1 expression in serum of PTC is downregulated and plays a role in the occurrence of PTC, which is able to guide the diagnosis and treatment in clinical practice.

\section{Acknowledgements}

Not applicable.

\section{Funding}

No funding was received.

\section{Availability of data and materials}

The datasets used and/or analyzed during the current study are available from the corresponding author on reasonable request.

\section{Authors' contributions}

TZ contributed to the conception and design of the study and drafted the study. HW was responsible for acquisition and analysis of data and revised the manuscript . Both authors read and approved the final manuscript.

\section{Ethics approval and consent to participate}

The study was approved by the Ethics Committee of the Second Affiliated Hospital of Soochow University (Suzhou, China). Signed written informed consents were obtained from the patients.

\section{Consent for publication}

Not applicable.

\section{Competing interests}

The authors declare that they have no competing interests.

\section{References}

1. Valmasoni M,Pierobon ES, Ruol A,De Pasqual CA,Zanchettin G, Moletta L, Salvador R, Costantini M and Merigliano S: Endoscopic tumor length should be reincluded in the esophageal cancer staging system: Analyses of 662 consecutive patients. PLoS One 11: e0153068, 2016.

2. Mazzaferri EL and Young RL: Papillary thyroid carcinoma: A 10 year follow-up report of the impact of therapy in 576 patients. Am J Med 70: 511-518, 1981.

3. Jazdzewski K, Murray EL, Franssila K, Jarzab B, Schoenberg DR and de la Chapelle A: Common SNP in pre-miR-146a decreases mature miR expression and predisposes to papillary thyroid carcinoma. Proc Natl Acad Sci USA 105: 7269-7274, 2008.

4. Huang Y, Prasad M, Lemon WJ, Hampel H, Wright FA, Kornacker K, LiVolsi V, Frankel W, Kloos RT, Eng C, et al: Gene expression in papillary thyroid carcinoma reveals highly consistent profiles. Proc Natl Acad Sci USA 98: 15044-15049, 2001.

5. Hu Y, Wang H, Chen E, Xu Z, Chen B and Lu G: Candidate microRNAs as biomarkers of thyroid carcinoma: A systematic review, meta-analysis, and experimental validation. Cancer Med 5: 2602-2614, 2016.

6. Agrawal N, Akbani R, Aksoy BA, Ally A, Arachchi H, Asa SL, Auman JT, Balasundaram M, Balu S, Baylin SB, et al; Cancer Genome Atlas Research Network: Integrated genomic characterization of papillary thyroid carcinoma. Cell 159: 676-690, 2014.

7. Fu XM, Guo W, Li N, Liu HZ, Liu J, Qiu SQ, Zhang Q, Wang LC, $\mathrm{Li} \mathrm{F}$ and Li CL: The expression and function of long noncoding RNA lncRNA-ATB in papillary thyroid cancer. Eur Rev Med Pharmacol Sci 21: 3239-3246, 2017.

8. Ragazzi M, Ciarrocchi A, Sancisi V, Gandolfi G, Bisagni A and Piana S: Update on anaplastic thyroid carcinoma: Morphological, molecular, and genetic features of the most aggressive thyroid cancer. Int J Endocrinol 2014: 790834, 2014.

9. Wei WJ, Lu ZW, Wang Y, Zhu YX, Wang YL and Ji QH: Clinical significance of papillary thyroid cancer risk loci identified by genome-wide association studies. Cancer Genet 208: 68-75, 2015.

10. Liu X, Qu S, Liu R, Sheng C, Shi X, Zhu G, Murugan AK, Guan $\mathrm{H}$, Yu H, Wang Y, et al: TERT promoter mutations and their association with BRAF V600E mutation and aggressive clinicopathological characteristics of thyroid cancer. J Clin Endocrinol Metab 99: E1130-E1136, 2014.

11. Kunstman JW, Juhlin CC, Goh G, Brown TC, Stenman A, Healy JM, Rubinstein JC, Choi M, Kiss N, Nelson-Williams C, et al: Characterization of the mutational landscape of anaplastic thyroid cancer via whole-exome sequencing. Hum Mol Genet 24: 2318-2329, 2015

12. Wang L, Mo X, Xu Y, Zuo B, Lei M, Li F, Jiang S, Deng C and Xiong Y: Molecular characterization and expression patterns of AMP deaminase1 (AMPD1) in porcine skeletal muscle. Comp Biochem Physiol B Biochem Mol Biol 151: 159-166, 2008.

13. Tousoulis D, Kioufis S, Siasos G, Oikonomou E, Zaromitidou M, Maniatis K, Kokkou E, Mazaris S, Zakynthinos G, Konsola T, et al: The impact of AMPD1 gene polymorphism on vascular function and inflammation in patients with coronary artery disease. Int J Cardiol 172: e516-e518, 2014. 
14. Sabina RL, Morisaki T, Clarke P, Eddy R, Shows TB, Morton CC and Holmes EW: Characterization of the human and rat myoadenylate deaminase genes. J Biol Chem 265: 9423-9433, 1990.

15. Fischer S, Drenckhahn C, Wolf C, Eschrich K, Kellermann S, Froster UG and Schober R: Clinical significance and neuropathology of primary MADD in C34-T and G468-T mutations of the AMPD1 gene. Clin Neuropathol 24: 77-85, 2005.

16. Loh E, Rebbeck TR, Mahoney PD, DeNofrio D, Swain JL and Holmes EW: Common variant in AMPD1 gene predicts improved clinical outcome in patients with heart failure. Circulation 99: 1422-1425, 1999.

17. Li J, Yang T, Zhao T, Liang J and Lin YS: Clinical outcome of radioiodine therapy in low-intermediate risk papillary thyroid carcinoma with BRAF(V600E) mutation. Zhongguo Yi Xue Ke Xue Yuan Xue Bao 38: 346-350, 2016 (In Chinese).

18. Cheng J, Morisaki H, Toyama K, Sugimoto N, Shintani T, Tandelilin A, Hirase T, Holmes EW and Morisaki T: AMPD1: A novel therapeutic target for reversing insulin resistance. BMC Endocr Disord 14: 96, 2014.
19. Feng AF, Liu ZH, Zhou SL, Zhao SY, Zhu YX and Wang HX: Effects of AMPD1 gene C34T polymorphism on cardiac index, blood pressure and prognosis in patients with cardiovascular diseases: A meta-analysis. BMC Cardiovasc Disord 17: 174, 2017.

20. Hay ID, Bergstralh EJ, Goellner JR, Ebersold JR and Grant CS: Predicting outcome in papillary thyroid carcinoma: Development of a reliable prognostic scoring system in a cohort of 1779 patients surgically treated at one institution during 1940 through 1989. Surgery 114: 1050-1058, 1993.

This work is licensed under a Creative Commons Attribution-NonCommercial-NoDerivatives 4.0 International (CC BY-NC-ND 4.0) License. 